\title{
Multi-response optimization of Ti-6Al-4V turning operations using Taguchi-based grey relational analysis coupled with kernel principal component analysis
}

\author{
Ning $\mathrm{Li}^{1} \cdot$ Yong-Jie Chen ${ }^{1} \cdot$ Dong-Dong Kong ${ }^{1}$
}

Received: 21 August 2018/Revised: 7 October 2018/Accepted: 19 March 2019/Published online: 9 May 2019

(C) The Author(s) 2019

\begin{abstract}
Ti-6Al-4V has a wide range of applications, especially in the aerospace field; however, it is a difficultto-cut material. In order to achieve sustainable machining of Ti-6Al-4V, multiple objectives considering not only economic and technical requirements but also the environmental requirement need to be optimized simultaneously. In this work, the optimization design of process parameters such as type of inserts, feed rate, and depth of cut for Ti-6Al-4V turning under dry condition was investigated experimentally. The major performance indexes chosen to evaluate this sustainable process were radial thrust, cutting power, and coefficient of friction at the toolchip interface. Considering the nonlinearity between the various objectives, grey relational analysis (GRA) was first performed to transform these indexes into the corresponding grey relational coefficients, and then kernel principal component analysis (KPCA) was applied to extract the kernel principal components and determine the corresponding weights which showed their relative importance. Eventually, kernel grey relational grade (KGRG) was proposed as the optimization criterion to identify the optimal combination of process parameters. The results of the range analysis show that the depth of cut has the most significant effect, followed by the feed rate and type of inserts. Confirmation tests clearly show that the modified method combining GRA with KPCA outperforms the traditional GRA method with equal weights and the hybrid method based on GRA and PCA.
\end{abstract}

Yong-Jie Chen

yjchen@hust.edu.cn

1 School of Mechanical Science and Engineering, Huazhong University of Science and Technology, Wuhan 430074, People's Republic of China
Keywords Ti-6Al-4V · Taguchi method · Grey relational analysis (GRA) · Kernel principal component analysis $($ KPCA $) \cdot$ Multi-response optimization

\section{Introduction}

Because of their many excellent properties, such as high strength-to-weight ratio and good heat and corrosion resistance, titanium alloys have a wide range of applications, especially in the aerospace field with about $50 \%$ of the world's total titanium [1]. Among them, one of the most commonly used is Ti-6Al-4V. However, this alloy is difficult to cut because of its low thermal conductivity and high chemical reactivity, which leads to rapid tool wear or breakage. About $80 \%$ of the heat generated during the cutting process is conducted into the cutting tool, which greatly weakens its cutting performance [2]. In order to improve the poor machinability of Ti-6Al-4V and reduce the machining cost, one way is to develop new tool materials to replace the commonly used cemented carbide [3], such as polycrystalline diamond (PCD) [4] and polycrystalline cubic boron nitride (PCBN) [5], which can improve the machinability of Ti-6Al-4V effectively. However, it is too expensive to be considered. Another way is to improve the tool structure, such as introducing friction-reducing grooves [6] or surface textures $[7,8]$ on the tool face, which can reduce cutting forces, decrease cutting temperature, and improve the friction condition at the toolchip interface, thus enhancing tool life. However, surface texturing is often fabricated on inserts with a flat rake face or flank face, which is not commonly used. Research on the application of surface texturing to improve cutting performance of inserts with 3D complex topography has rarely been reported. Therefore, it is necessary to apply surface 
texturing to inserts with a $3 \mathrm{D}$ shape on the rake face to improve the machinability of Ti-6Al-4V. The most effective and advisable way is to optimize the existing process parameters, which requires lower investment and has better social sustainability without making drastic changes. In addition, it is most likely to be accepted by users [9, 10]. In the present work, the process parameters, such as type of inserts (with texture or not), feed rate, and depth of cut, were optimized to improve the machinability of Ti-6Al-4V.

In the machining process, common performance indexes for evaluating machinability include productivity, operating cost, and product quality [11]. Because of the inconvenience of direct measurement, they are usually described by measurable process outputs, such as tool life, surface roughness, cutting forces, power consumption, and vibration. Today, many studies focus on the optimization of individual performance characteristic in the machining processes. Subramanian et al. [12] developed a statistical model to envisage vibration amplitude in terms of process parameters such as radial rake angle, tool nose radius, cutting speed, feed rate, and axial depth of cut by response surface methodology (RSM) during end milling of $\mathrm{Al}$ 7075-T6. They found that the vibration amplitude was maintained at the minimum under the conditions of a radial rake angle of $12^{\circ}$, nose radius of $0.8 \mathrm{~mm}$, cutting speed of $115 \mathrm{~m} / \mathrm{min}$, feed rate of $0.16 \mathrm{~mm} / \mathrm{r}$, and axial depth of cut of $2.5 \mathrm{~mm}$. By combining genetic algorithms (GA) and RSM, Singh and Rao [13] obtained the optimum machining conditions of a cutting speed of $200 \mathrm{~m} / \mathrm{min}$, feed rate of 0.1 $\mathrm{mm} / \mathrm{r}$, effective rake angle of $6^{\circ}$, and nose radius of $1.2 \mathrm{~mm}$ for a minimum surface roughness value of $0.4866 \mu \mathrm{m}$ during hard turning of AISI 52100 steel. Ramana et al. [14] concluded that the optimal process parameters for minimizing tool wear were an MQL machining environment, cutting speed of $63 \mathrm{~m} / \mathrm{min}$, feed rate of $0.274 \mathrm{~mm} / \mathrm{r}$, depth of cut of $1.0 \mathrm{~mm}$, and uncoated tool during turning of Ti$6 \mathrm{Al}-4 \mathrm{~V}$ by Taguchi's robust design methodology. In short, all of these studies have achieved good results without considering other indexes. However, in practice, the optimization of the individual performance index is often achieved at the cost of deteriorating other indexes due to the complexity and conflict between the indexes. For example, a higher cutting speed helps increase productivity and surface quality, but also accelerates tool wear, leading to shorter tool life and increased production costs. Therefore, in order to achieve sustainable manufacturing, especially for titanium alloys, it is more reasonable to optimize multiple responses simultaneously based on environmentally sustainable, economic, and technical requirements.

Related research on multi-response optimization in the cutting process, considering several contradictory responses, has also been frequently reported. Yan and Li [15] optimized cutting parameters such as spindle speed, feed rate, depth of cut, and width of cut in the milling process based on weighted grey relational analysis (GRA) and RSM in order to evaluate trade-offs between sustainability, production rate, and cutting quality. Surface roughness, material removal rate, and cutting energy were evaluated simultaneously. Sarkkaya and Güllü [16] optimized the process parameters such as cutting fluid, fluid flow rate, and cutting speed by Taguchi based GRA, taking flank wear, notch wear, and surface roughness as process performance indexes. In order to minimize surface roughness and burr formation concurrently, a multi-objective particle swarm optimization method was utilized to find the optimal cutting parameters during micro-end milling of Ti-6Al-4V by Thepsonthi and Özel [17]. Yi et al. [18] presented a multi-objective optimization model based on a non-dominated sorting GA to explore the impact of cutting speed and feed rate on carbon emissions and processing time. To obtain favorable performance characteristics such as material removal rate, cutting force, and surface roughness during dry turning of AISI 304 austenitic stainless steel, Nayak et al. [19] combined Taguchi method and GRA to optimize the machining parameters such as cutting speed, feed rate, and depth of cut. Mia et al. [20] first attempted to optimize cutting forces, surface roughness, cutting temperature, and chip reduction coefficient during turning of Ti-6Al-4V under dry and high-pressure coolant using GRA integrated with Taguchi method. Afterwards, they experimentally investigated surface roughness, cutting force, and feed force during turning of Ti-6Al-4V under cryogenic (liquid nitrogen) condition and performed the desirabilitybased multi-response optimization according to the models of responses by RSM and artificial neural network [21].

All the above literatures have achieved the multi-objective optimization that simultaneously meets the environment sustainable, economic, and technical requirements by different indexes and methods. At the same time, it also shows the complexity and diversity of indexes. In the present work, performance indexes such as radial thrust, cutting power, and coefficient of friction at the tool-chip interface were chosen to evaluate the machinability of Ti$6 \mathrm{Al}-4 \mathrm{~V}$ for the following reasons. Firstly, in spite of not doing any work, radial thrust may lead to deformation or vibration of the workpiece, which will greatly increase the likelihood of tool breakage and directly affect machining accuracy and surface quality, thereby indirectly increasing the processing costs [22]. Therefore, it can be taken as a technical or economic index. Besides, research on the use of radial thrust as an optimization index has rarely been reported. Secondly, the contradiction between the growing demand for energy and the increasing severity of resource shortage, along with more and more serious environmental problems, forces us to pay more attention to energy conservation and emissions reduction, especially in 
manufacturing. Energy savings can reach up to $6 \%-40 \%$ by selecting the optimal combination of process parameters [23]. Power consumption is a very important index reflecting energy consumption during the cutting process, which can be derived from the measured tangential force and axial thrust. Because more difficult-to-cut materials will require more power and greater cutting force or torque [11], power consumption can be considered as an environment sustainable or technical index. Thirdly, coefficient of friction at the tool-chip interface is influenced by many factors such as work material, tool material, tool geometry, cutting parameters, and cutting fluid. However, it has a great influence on many other indexes such as energy consumption and tool life. The larger the coefficient of friction at the tool-chip interface is, the more energy will be consumed and the more severe tool wear will appear. Hence, it can be regarded as an environment sustainable or economic index. Furthermore, little research has been reported about taking coefficient of friction at the tool-chip interface as an optimization index for Ti-6Al-4V turning according to the previous researches. The only relevant study by Mia et al. [24] was reported not long ago, which focused on the optimization of chip compression ratio, effective shear angle, friction coefficient at the tool rake surface and the chip-tool interface temperature in turning process using grey relation-based Taguchi method. At last, these three indexes are easy to obtain based on the acquired cutting force signals.

Based on the implementation method of the optimization procedure, the multi-objective optimization method can be divided into the priori $[15,16,19]$ and posterior technique $[17,18]$. Due to the advantages of small number of tests, simple calculations, and unique optimal results, the priori technique like Taguchi-based GRA has always been favored by many scholars, whose basic idea is to transform the multi-objective optimization problem into a single objective optimization problem [25]. However, a key question exists that the weights of multiple performance indexes on the single objective are hard to determine. In many literatures, equal weights $[16,26]$ or weights assigned by decision makers $[2,27]$ are often applied to describe the relative importance of each performance index, which results in great uncertainty and irrationality. In order to solve this problem, many scholars have devoted much attention and made some achievements. Lu et al. [28] performed the principal component analysis (PCA) on the grey relational coefficients (GRC) corresponding to various performance indexes, and extracted the square values of three components in the first principal component as the weighting values. Dubey and Yadava [29] applied a hybrid approach based on the Taguchi method and PCA for multiobjective optimization by extracting three principal components from the three quality characteristics, and then transforming into a unique total principal component index with the proportion of each eigenvalue as weighting value. It is well known that the basic principle of PCA is to apply the linear combinations of the original correlation variables to represent the main characteristics of the objectives, which is a method to solve multicollinearity. However, the relationship between various performance indexes in the cutting process tends to be nonlinear, so it is no longer appropriate to use PCA to deal with this multi-response optimization problem. Because kernel principal component analysis (KPCA) is a nonlinear extension of PCA using kernel technique, KPCA can be applied to solve nonlinear problems in multi-response optimization.

Taking the nonlinearity of cutting process into consideration, a hybrid method based on GRA and KPCA was proposed for solving the multi-response optimization problem during turning of Ti-6Al-4V in the present work. The major performance indexes chosen to evaluate this sustainable process were radial thrust, cutting power, and coefficient of friction at the tool-chip interface. The structure of this work was arranged as follows: Firstly, the optimization methods were presented in detail, and Taguchi's orthogonal array (OA) $L_{16}$ with mixed levels was designed to obtain the performance indexes. Secondly, the optimization procedure based on GRA and KPCA was described step-by-step. Thirdly, the effect of process parameters such as type of inserts, feed rate, and depth of cut on the optimization criterion was investigated. At last, the optimal combination of process parameters was verified and the conclusion was drawn. The workflow diagram of multi-response optimization is shown in Fig. 1.

\section{Methodology and experimental design}

\subsection{Taguchi method}

Taguchi method is an ideal method to improve product quality, promote technological innovation, and increase the competitiveness of enterprises, which is helpful to develop low-cost and high-quality products within a relatively short time. To quantitatively describe the product quality loss, the concept of "quality loss function" is put forward and the signal-to-noise ratio $(S / N)$ for each quality characteristic is applied to evaluate the robustness of the designed process parameters. There are three types of quality characteristics: the lower the better, the higher the better, and the nominal the better. In terms of radial thrust, power consumption, and coefficient of friction at the tool-chip interface, $S / N$ with a lower-the-better characteristic [30] is desirable, which can be expressed as 


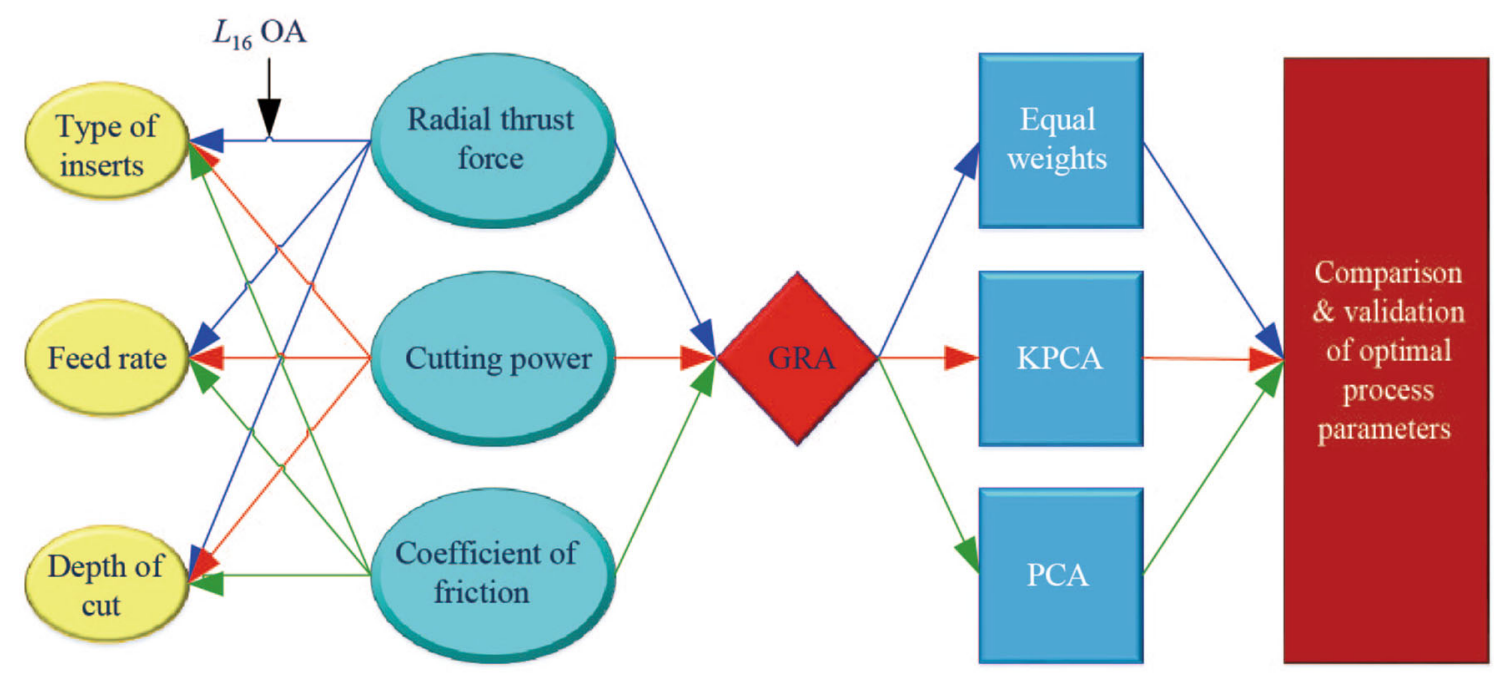

Fig. 1 Workflow diagram of multi-response optimization

$\eta_{i}(k)=-10 \lg \left(\frac{1}{n} \sum_{j=1}^{n} y_{i j}^{2}(k)\right)$

where $y_{i j}(k)$ is the observed data of the $k$ th performance index at the $i$ th experiment at the $j$ th trial; $i=1,2, \cdots, m ; j=$ $1,2, \cdots, n ; k=1,2, \cdots, s$; and $n$ is the number of repetitions of the same experiment. In this paper, $m=16, n=1, s=$ 3.

\subsection{Principle of GRA}

Grey system theory is a systematic scientific theory proposed by Deng in Ref. [31] to solve the problems with insufficient, poor, or uncertain information. Actually, the optimization problem of multiple performance indexes with the lower-the-better characteristics itself contains a certain degree of uncertainty and ambiguity. Therefore, the grey system theory is very suitable for solving this multiresponse optimization problem. Based on this theory, GRA is carried out to evaluate the degree of correlation between the observed data (comparability sequence) and the desired value (reference sequence), and transform multiple performance characteristics into a single grey relational grade (GRG) value as the optimization criterion.

In order to avoid the inconvenience caused by different dimensions in the observed data and simplify the calculation, the first step for GRA is grey relational generating [31], that is, to normalize these observed data to the range $[0,1]$. Because $S / N$ refers to the ratio between the desired part and the noise part, the larger $S / N$ is the better [29], regardless of the nature of quality characteristics. Therefore, in this paper, $S / N$ for each quality characteristic is normalized by the following equation $\eta_{i}^{*}(k)=\frac{\eta_{i}(k)-\min \eta_{i}(\mathrm{k})}{\max \eta_{i}(k)-\min \eta_{i}(k)}$,

where $\eta_{i}^{*}(k)$ is the $k$ th comparability sequence after the process of grey relational generating; $\max \eta_{i}(k)$ and $\min \eta_{i}(k)$ are the maximum and minimum values of the $k$ th sequence of $S / N$. Due to the fact that the larger normalized value corresponds to the better performance, the maximum normalized value is considered as the reference sequence, denoted by $\eta_{\mathrm{o}}^{*}(k)=1$.

Then, the relationship between the observed data and the desired value can be clarified clearly by calculating the GRC, $\xi$, between the comparability sequences and the reference sequence. The $\xi$ for the $k$ th performance index at the $i$ th experiment can be expressed as

$\xi_{i}(k)=\frac{\min _{i} \min _{k} \Delta_{i}(k)+\rho \max _{i} \max _{k} \Delta_{i}(k)}{\Delta_{i}(k)+\rho \max _{i} \max _{k} \Delta_{i}(k)}$,

where $\Delta_{i}(k)=\left|\eta_{\mathrm{o}}^{*}(k)-\eta_{i}^{*}(k)\right|$ is the $k$ th deviation sequence in absolute value between the reference sequence and the $k$ th comparability sequence; distinguishing coefficient $\rho \in(0,+\infty)$ is a factor affecting the resolution of correlation analysis directly, which determines the distribution of GRC. The smaller the distinguishing coefficient, the greater the resolution. Usually, $\rho=0.5$ can meet the demand and is adopted in this paper.

At last, a single GRG value, $\gamma$, for the $i$ th experiment can be obtained by a weighted sum of the above GRC with the following formula

$\gamma(i)=\sum_{k=1}^{s} \omega_{k} \xi_{i}(k)$, 
where $\omega_{k}$ denotes the weight of the $k$ th performance index, which meets $\sum_{k=1}^{s} \omega_{k}=1$. Usually, equal weights are artificially set. The higher $\gamma$ indicates the closer relational degree between the observed data and the desired value, which means that the corresponding combination of process parameters is closer to the optimal. At this point, the multi-response optimization problem has been fully transformed into a single objective optimization problem with $\gamma$ as the optimization criterion.

However, due to the nonlinearity between the performance indexes, this optimization criterion is not very reasonable. In order to reveal the principal features of these performance indexes, KPCA will be introduced and an improved $\gamma$ will be proposed, with the corresponding weights also obtained in the next section.

\subsection{Principle of KPCA}

As mentioned earlier, PCA reported by Dubey and Yadava [29] and Lu et al. [28] is a linear mapping method that ignores the correlation of higher than the second order between the performance indexes. Therefore, the extracted principal features are not optimal, and the effect of PCA is affected to some extent. To solve this problem, KPCA is adopted in this paper. As a nonlinear expansion algorithm of PCA, KPCA uses the kernel-based technique to extract the principal components. That is to say, the principle of KPCA is that: at first, map the original vector to the highdimensional kernel space $F$ through mapping function $\psi$, and then perform PCA. Here, the original vector refers to the above GRC for each performance index. The specific implementation steps for KPCA are presented as

(i) Constituting the original performance index array $\xi_{i}(k), i=1,2, \cdots, m ; k=1,2, \cdots, s$

$$
\boldsymbol{\Xi}=\left[\begin{array}{cccc}
\xi_{1}(1) & \xi_{1}(2) & \cdots & \xi_{1}(s) \\
\xi_{2}(1) & \xi_{2}(2) & \cdots & \xi_{2}(s) \\
\vdots & \vdots & \vdots & \vdots \\
\xi_{m}(1) & \xi_{m}(2) & \cdots & \xi_{m}(s)
\end{array}\right]
$$

where $m$ and $s$ are the number of experiments and performance indexes, respectively.

(ii) Selecting the kernel function $\psi$ and calculating the kernel matrix $\boldsymbol{K}$

$$
\begin{aligned}
K_{\mu v} & =\boldsymbol{K}\left(\boldsymbol{\Xi}_{\mu}, \boldsymbol{\Xi}_{v}\right)=\left\langle\psi\left(\boldsymbol{\Xi}_{\mu}\right), \psi\left(\boldsymbol{\Xi}_{v}\right)\right\rangle \\
& =\exp \left(-\left\|\boldsymbol{\Xi}_{\mu}-\boldsymbol{\Xi}_{v}\right\|^{2} /\left(2 \sigma^{2}\right)\right),
\end{aligned}
$$

where $\boldsymbol{\Xi}_{\mu}$ and $\boldsymbol{\Xi}_{v}$ denote the performance index vector at the $\mu$ th and $v$ th experiment; $\mu=$ $1,2, \cdots, m ; v=1,2, \cdots, m$. In this paper,
Gaussian radial basis function is selected as the kernel function.

(iii) Centering the kernel matrix $\boldsymbol{K}$

$$
\begin{aligned}
K_{\mu \nu}^{*}= & K_{\mu \nu}-\frac{1}{m}\left(\sum_{\omega=1}^{m} K_{\mu \omega}+\sum_{\tau=1}^{m} K_{\tau v}\right) \\
& +\frac{1}{m^{2}} \sum_{\omega, \tau=1}^{m} K_{\omega \tau} .
\end{aligned}
$$

(iv) Determining the eigenvalues and eigenvectors from the above centered matrix $\boldsymbol{K}^{*}$

$\lambda_{i}^{*} \boldsymbol{\alpha}_{i}=\boldsymbol{K}^{*} \boldsymbol{\alpha}_{i}$

where $\lambda_{i}^{*}$ is the $i$ th eigenvalue, $i=1,2, \cdots, m$; $\boldsymbol{\alpha}_{i}=\left(\alpha_{i 1}, \alpha_{i 2}, \cdots, \alpha_{i m}\right)^{\mathrm{T}}$ is the corresponding eigenvector. Assuming that $\lambda_{1}^{*} \geqslant \lambda_{2}^{*} \geqslant \cdots \geqslant \lambda_{m}^{*}$, and the eigenvectors are normalized.

(v) Calculating the contribution rate $c(k)$ of the first $s$ eigenvalues as the weight of the performance index and extracting the corresponding kernel principal components $p_{i}(k)$

$$
\begin{aligned}
& c(k)=\lambda_{k}^{*} / \sum_{i=1}^{m} \lambda_{i}^{*}, \\
& p_{i}(k)=\sum_{j=1}^{s} \xi_{i}(j) \alpha_{k j} .
\end{aligned}
$$

All the eigenvalues are arranged in descending order with respect to variance. Usually, the accumulative contribution rate of the first three eigenvalues reaches over $90 \%$, which means the first three kernel principal components hold the most amount of information in the data. Therefore, the first three eigenvalues are taken into consideration in this work.

At last, the proposed new optimization criterion, $\gamma_{k}$ can be constructed as

$\gamma_{k}(i)=\sum_{k=1}^{s}\left|p_{i}(k)\right| c(k)$.

Based on this optimization criterion, the effect of each factor at different levels can be evaluated, and the optimum parameter combination corresponding to the maximum $\gamma_{k}$ is obtained, which can be compared with that obtained from $\gamma$.

\subsection{Design of experiment and test results}

All the tests were conducted on a CNC horizontal turning center (QTN200M/1000U) under dry condition. Titanium alloy Ti-6Al-4V bars with the dimension of $\phi 100-130 \mathrm{~mm}$ were used as workpiece material, and the tungsten carbide 


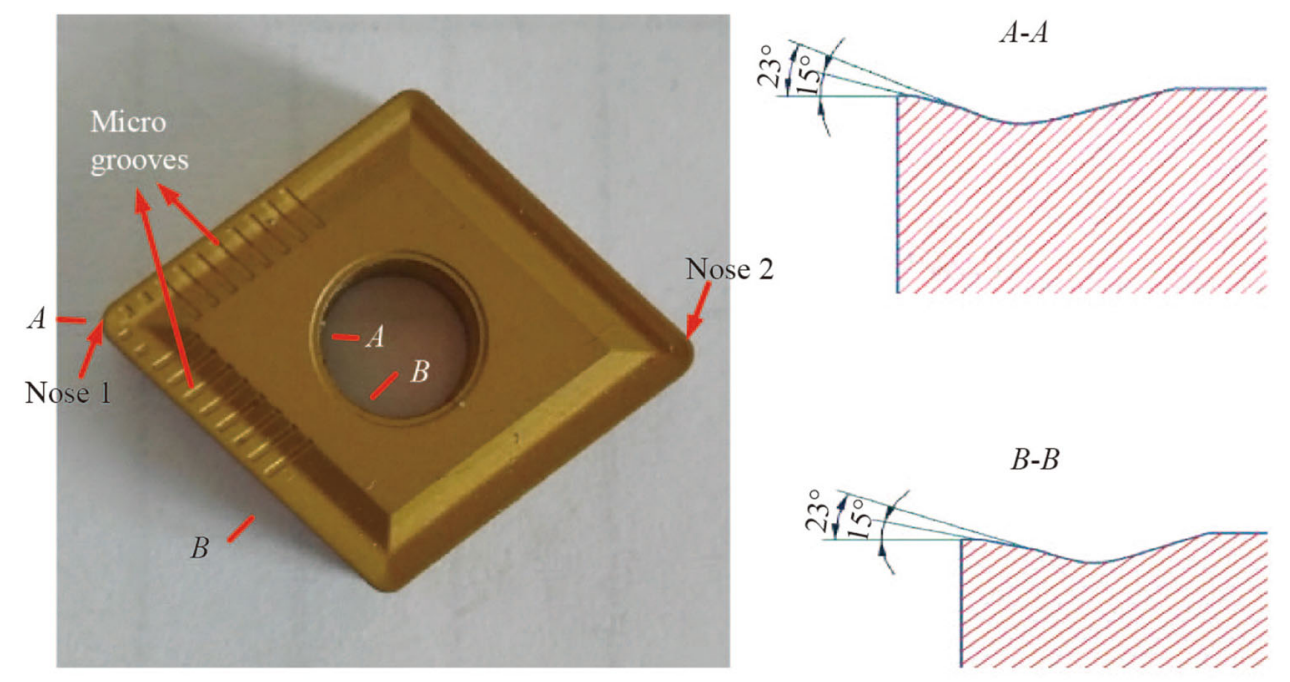

Fig. 2 Inserts used in the experiments

Table 1 Process parameters and their levels in the tests

\begin{tabular}{llllll}
\hline Process parameter & Symbol & Level 1 & Level 2 & Level 3 & Level 4 \\
\hline Type of inserts & $A$ & Nose 1 & Nose 2* & 0.20 & 0.25 \\
Feed rate/ $\left(\mathrm{mm} \cdot \mathrm{r}^{-1}\right)$ & $B$ & 0.10 & $0.15^{*}$ & 1.5 & 2.0 \\
Depth of cut/ mm & $C$ & 0.5 & $1.0^{*}$ & \\
\hline
\end{tabular}

*Initial process condition

inserts with grade $K$ were employed as cutting tools with the ISO designation of CNMG120408-JLPM1 (see Fig. 2), mounted on a PCLNR 2525M 12 tool holder. Since the performance indexes chosen in the present work depend on the process parameters such as type of inserts, cutting speed, feed rate, and depth of cut, they all can be selected as the controllable factors. However, because Ti- $6 \mathrm{Al}-4 \mathrm{~V}$ is a type of difficult-to-cut material, the higher cutting speed will aggravate tool wear. Therefore, a constant cutting speed, $V_{\mathrm{c}}=50 \mathrm{~m} / \mathrm{min}$, is used in the tests. As for feed rate and depth of cut, four different levels were selected from the manufacturer's recommended ranges $\left(V_{\mathrm{c}}=30-60 \mathrm{~mm} /\right.$ $\left.\min , f=0.05-0.35 \mathrm{~mm} / \mathrm{r}, a_{\mathrm{p}}=0.07-3 \mathrm{~mm}\right)$. In order to improve the friction property at the tool-chip interface, parallel array grooves were symmetrically fabricated on both sides of the tool tip, the direction of which formed an $80^{\circ}$ angle with the cutting edge, as shown in Fig. 2. The parameters of these grooves were a width of $0.3 \mathrm{~mm}$, depth of $0.05 \mathrm{~mm}$, and interval of $0.4 \mathrm{~mm}$. In the present work, two kinds of inserts were employed: one with these grooves named "Nose 1", and the other without grooves called "Nose 2". All the selected process parameters and their levels are listed in Table 1. Traditionally, the desired process parameters were mainly determined based on experience or specialized handbooks, which were not the
Table 2 Experimental layout using $L_{16}\left(2^{1} \times 4^{2}\right)$ and their responses

\begin{tabular}{|c|c|c|c|c|c|c|}
\hline \multirow[t]{2}{*}{ Run No. } & \multicolumn{3}{|c|}{ Factors and their levels } & \multirow[t]{2}{*}{$F_{y} / \mathrm{N}$} & \multirow[t]{2}{*}{$P / \mathrm{W}$} & \multirow[t]{2}{*}{$\mu$} \\
\hline & $A$ & $B$ & $C$ & & & \\
\hline 1 & 1 & 1 & 1 & 160.09 & 85.80 & 2.20 \\
\hline 2 & 1 & 2 & 2 & 225.33 & 208.49 & 2.12 \\
\hline 3 & 1 & 3 & 3 & 245.72 & 409.87 & 1.92 \\
\hline 4 & 1 & 4 & 4 & 274.12 & 577.59 & 2.03 \\
\hline 5 & 1 & 1 & 2 & 177.10 & 200.72 & 1.75 \\
\hline 6 & 1 & 2 & 1 & 188.69 & 101.66 & 2.58 \\
\hline 7 & 1 & 3 & 4 & 253.26 & 553.70 & 1.84 \\
\hline 8 & 1 & 4 & 3 & 266.04 & 428.02 & 2.15 \\
\hline 9 & 2 & 1 & 3 & 169.67 & 341.47 & 1.46 \\
\hline 10 & 2 & 2 & 4 & 229.23 & 507.53 & 1.64 \\
\hline 11 & 2 & 3 & 1 & 200.63 & 107.58 & 2.89 \\
\hline 12 & 2 & 4 & 2 & 245.34 & 222.39 & 2.97 \\
\hline 13 & 2 & 1 & 4 & 181.15 & 481.27 & 1.37 \\
\hline 14 & 2 & 2 & 3 & 206.84 & 358.48 & 1.81 \\
\hline 15 & 2 & 3 & 2 & 223.52 & 215.21 & 2.52 \\
\hline 16 & 2 & 4 & 1 & 193.54 & 101.12 & 3.43 \\
\hline
\end{tabular}

optimal values [15]. In this work, the initial process parameters were $A_{2}, B_{2}$, and $C_{2}$; the type of inserts was Nose 2; the cutting speed was $50 \mathrm{~mm} / \mathrm{r}$; the feed rate 0.15 
$\mathrm{mm} / \mathrm{r}$ and the depth of cut $1.0 \mathrm{~mm}$, per the manufacturer's recommendations, before the parallel array grooves were fabricated on the tool rake face.

As can be seen from Table 1, the total degree of freedom of the process parameters is 7 in the case of ignoring the interactions. Therefore, based on the Taguchi's robust design concept, an OA with mixed levels $L_{16}\left(2^{1} \times 4^{2}\right)$ was redesigned by transforming the standard $L_{16}\left(2^{15}\right)$ OA to reduce machining time and cost. The resulting experimental layout was presented in Table 2. In this study, radial thrust $\left(F_{y}\right)$, power consumption $(P)$, and coefficient of friction $(\mu)$ at the tool-chip interface are chosen as research indexes to assess the machining performance; $F_{y}$ is obtained by direct measurement, whereas $P$ and $\mu$ can be derived from the following formulas $[5,22]$

$P=F_{z} V_{\mathrm{c}} / 60+F_{x} n_{\mathrm{w}} f / 60000$

$\mu=\frac{\left|\left\{F_{x}, F_{y}, F_{z}\right\} \times\left\{\cos \gamma_{\mathrm{p}} \sin \gamma_{\mathrm{f}}, \sin \gamma_{\mathrm{p}} \cos \gamma_{\mathrm{f}},-\cos \gamma_{\mathrm{p}} \cos \gamma_{\mathrm{f}}\right\}\right|}{\left|\left\{F_{x}, F_{y}, F_{z}\right\} \cdot\left\{\cos \gamma_{\mathrm{p}} \sin \gamma_{\mathrm{f}}, \sin \gamma_{\mathrm{p}} \cos \gamma_{\mathrm{f}},-\cos \gamma_{\mathrm{p}} \cos \gamma_{\mathrm{f}}\right\}\right|}$,

where $f$ is the feed rate, $\mathrm{mm} / \mathrm{r} ; n_{\mathrm{w}}$ is the rotating speed of the workpiece, $\mathrm{r} / \mathrm{min}$; and $\gamma_{\mathrm{p}}=-7.31^{\circ}, \gamma_{\mathrm{f}}=9.05^{\circ}$ are the back rake angle and side rake angle, respectively. Because they are functions of cutting forces $\left(F_{x}, F_{y}\right.$, and $\left.F_{z}\right)$, they are also relatively easy to obtain.

In order to measure the cutting forces under each run, a three-component piezoelectric dynamometer (Kistler 9257A) equipped with a charge amplifier (Kistler 5019) was applied, which was linked to the national instruments PXI system (PXI-1042Q/8106) for data acquisition. At last, the results were displayed on the monitor. The detailed experimental setup was schematically illustrated in Fig. 3. Besides, a constant cutting length along the feed direction, $L=5 \mathrm{~mm}$, was kept under each run to ignore the influence of tool wear. According to the cutting forces measured in

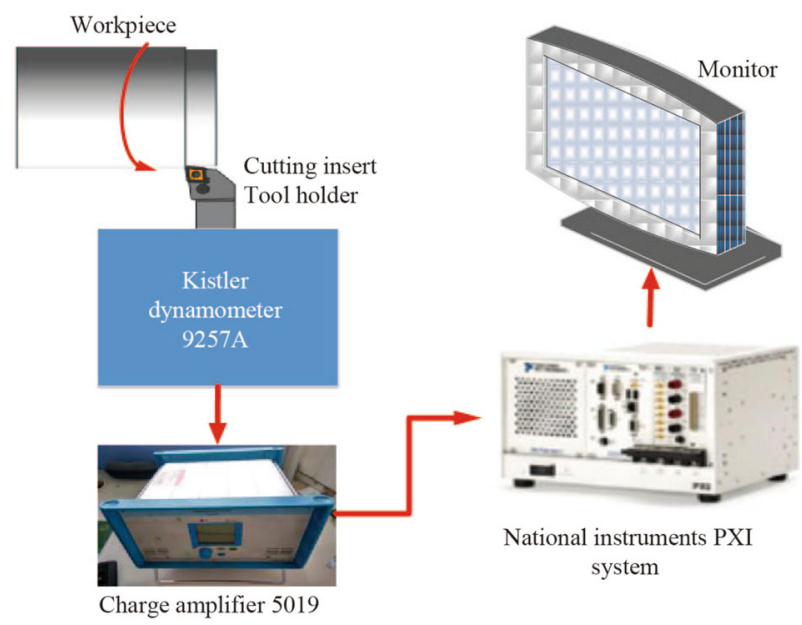

Fig. 3 Schematic diagram of the experimental setup the tests and the above Eqs. (12) and (13), the power consumption and the coefficient of friction at the tool-chip interface were calculated, and all the performance indexes are shown in Table 2.

\section{Results and discussion}

In this section, the approach based on GRA and KPCA to optimize the process parameters for Ti-6Al-4V turning is presented in detail, and the multi-response optimization results by $\gamma$, the integrated optimization criterion $\gamma_{\mathrm{p}}$, and $\gamma_{k}$ are compared and validated through the confirmation tests. Here, $\gamma_{p}$ denotes the corresponding grey relational grade obtained from the hybrid method based on GRA and PCA, which is used to illustrate the effect of nonlinearity.

\subsection{Detailed optimization procedure}

At first, data preprocessing was conducted on the performance indexes $F_{y}, P$, and $\mu$ obtained from the tests. Due to the lower-the-better characteristics, the $S / N$ of these performance indexes is calculated and normalized based on Eqs. (1) and (2), and the results are listed in Table 3. Thus, the comparability sequences are obtained. In Table 3, the values of the reference sequences are specified as 1, representing the best performance.

Table $3 S / N$ and its normalization for radial thrust force, cutting power and coefficient of friction

\begin{tabular}{|c|c|c|c|c|c|c|}
\hline \multirow[t]{2}{*}{ Run No. } & \multicolumn{3}{|c|}{$\begin{array}{l}S / N \text { ratios for different } \\
\text { responses }\end{array}$} & \multicolumn{3}{|c|}{$\begin{array}{l}\text { Normalization for different } \\
\text { responses }\end{array}$} \\
\hline & $F_{y}$ & $P$ & $\mu$ & $F_{y}$ & $P$ & $\mu$ \\
\hline 1 & -44.09 & -39.67 & -6.85 & 1.0000 & 1.0000 & 0.4835 \\
\hline 2 & -47.06 & -46.38 & -6.52 & 0.3645 & 0.5344 & 0.5250 \\
\hline 3 & -47.81 & -52.25 & -5.67 & 0.2034 & 0.1799 & 0.6320 \\
\hline 4 & -48.76 & -55.23 & -6.13 & 0.0000 & 0.0000 & 0.5745 \\
\hline 5 & -44.96 & -46.05 & -4.84 & 0.8123 & 0.5543 & 0.7361 \\
\hline 6 & -45.52 & -40.14 & -8.24 & 0.6944 & 0.9110 & 0.3095 \\
\hline 7 & -48.07 & -54.87 & -5.28 & 0.1472 & 0.0222 & 0.6816 \\
\hline 8 & -48.50 & -52.63 & -6.64 & 0.0557 & 0.1572 & 0.5103 \\
\hline 9 & -44.59 & -50.67 & -3.30 & 0.8920 & 0.2756 & 0.9306 \\
\hline 10 & -47.21 & -54.11 & -4.28 & 0.3325 & 0.0678 & 0.8074 \\
\hline 11 & -46.05 & -40.63 & -9.20 & 0.5803 & 0.8813 & 0.1883 \\
\hline 12 & -47.80 & -46.94 & -9.46 & 0.2063 & 0.5005 & 0.1565 \\
\hline 13 & -45.16 & -53.65 & -2.74 & 0.7703 & 0.0957 & 1.0000 \\
\hline 14 & -46.31 & -51.09 & -5.14 & 0.5237 & 0.2502 & 0.6985 \\
\hline 15 & -46.99 & -46.66 & -8.05 & 0.3795 & 0.5177 & 0.3338 \\
\hline 16 & -45.74 & -40.10 & -10.07 & 0.6472 & 0.9138 & 0.0000 \\
\hline \multicolumn{4}{|c|}{ Reference sequence } & 1.0000 & 1.0000 & 1.0000 \\
\hline
\end{tabular}


Table 4 Calculated $\Delta_{\mathrm{o} i}, \xi, \gamma$, and the corresponding ranking of $\gamma$

\begin{tabular}{|c|c|c|c|c|c|c|c|c|}
\hline \multirow[t]{2}{*}{ Run No. } & \multicolumn{3}{|c|}{ Deviation sequences } & \multicolumn{3}{|c|}{ Grey relational coefficient } & \multirow[t]{2}{*}{$\gamma$} & \multirow[t]{2}{*}{ Ranking } \\
\hline & $\Delta_{\mathrm{o} i}\left(F_{y}\right)$ & $\Delta_{\mathrm{o} i}(P)$ & $\Delta_{\mathrm{o} i}(\mu)$ & $\xi\left(F_{y}\right)$ & $\xi(P)$ & $\xi(\mu)$ & & \\
\hline 1 & 0.0000 & 0.0000 & 0.5165 & 1.0000 & 1.0000 & 0.4919 & 0.8306 & 1 \\
\hline 2 & 0.6355 & 0.4656 & 0.4750 & 0.4403 & 0.5178 & 0.5128 & 0.4903 & 10 \\
\hline 3 & 0.7966 & 0.8201 & 0.3680 & 0.3856 & 0.3788 & 0.5760 & 0.4468 & 12 \\
\hline 4 & 1.0000 & 1.0000 & 0.4255 & 0.3333 & 0.3333 & 0.5403 & 0.4023 & 16 \\
\hline 5 & 0.1877 & 0.4457 & 0.2639 & 0.7271 & 0.5287 & 0.6545 & 0.6368 & 4 \\
\hline 6 & 0.3056 & 0.0890 & 0.6905 & 0.6206 & 0.8489 & 0.4200 & 0.6299 & 5 \\
\hline 7 & 0.8528 & 0.9778 & 0.3184 & 0.3696 & 0.3383 & 0.6109 & 0.4396 & 13 \\
\hline 8 & 0.9443 & 0.8428 & 0.4897 & 0.3462 & 0.3723 & 0.5052 & 0.4079 & 15 \\
\hline 9 & 0.1080 & 0.7244 & 0.0694 & 0.8224 & 0.4084 & 0.8782 & 0.7030 & 2 \\
\hline 10 & 0.6675 & 0.9322 & 0.1926 & 0.4283 & 0.3491 & 0.7219 & 0.4998 & 9 \\
\hline 11 & 0.4197 & 0.1187 & 0.8117 & 0.5437 & 0.8082 & 0.3812 & 0.5777 & 7 \\
\hline 12 & 0.7937 & 0.4995 & 0.8435 & 0.3865 & 0.5003 & 0.3722 & 0.4196 & 14 \\
\hline 13 & 0.2297 & 0.9043 & 0.0000 & 0.6852 & 0.3560 & 1.0000 & 0.6804 & 3 \\
\hline 14 & 0.4763 & 0.7498 & 0.3015 & 0.5121 & 0.4000 & 0.6238 & 0.5120 & 8 \\
\hline 15 & 0.6205 & 0.4823 & 0.6662 & 0.4462 & 0.5090 & 0.4287 & 0.4613 & 11 \\
\hline 16 & 0.3528 & 0.0862 & 1.0000 & 0.5863 & 0.8530 & 0.3333 & 0.5909 & 6 \\
\hline
\end{tabular}

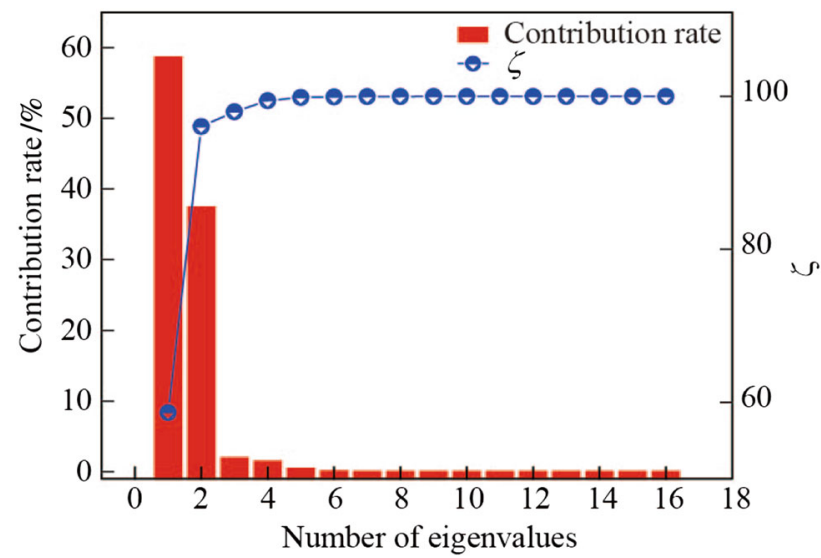

Fig. 4 Contribution rate of each eigenvalue and the accumulative contribution rate

Before calculating the GRC, the deviation sequences $\Delta_{\mathrm{o} i}\left(F_{y}\right), \Delta_{\mathrm{o} i}(P)$, and $\Delta_{\mathrm{o} i}(\mu)$ were determined, which denoted the degree that the observed values deviated from the expected value. Thus, according to Eq. (3), the GRC between the comparability sequences and the reference sequences were obtained and presented in Table 4, which constituted the original performance index array by Eq. (5) for KPCA in Section 2.3. In most cases, equal weights were assigned for different performance indexes in multiresponse optimization problems, with the interconnectedness between the performance indexes ignored. The resulting $\gamma$ was calculated by using Eq. (4). In this paper,
Table 5 The first three kernel principal components, $\gamma_{k}$ and the corresponding ranking of $\gamma_{k}$

\begin{tabular}{|c|c|c|c|c|c|}
\hline \multirow[t]{2}{*}{ Run No. } & \multicolumn{3}{|c|}{ Kernel principal components } & \multirow[t]{2}{*}{$\gamma_{k}$} & \multirow[t]{2}{*}{ Ranking } \\
\hline & First & Second & Third & & \\
\hline 1 & 0.2853 & -0.0924 & -0.0088 & 0.2063 & 1 \\
\hline 2 & -0.0181 & 0.0428 & 0.0016 & 0.0272 & 16 \\
\hline 3 & -0.0944 & 0.0361 & -0.0011 & 0.0703 & 11 \\
\hline 4 & -0.1147 & 0.0579 & -0.0034 & 0.0908 & 6 \\
\hline 5 & 0.0214 & -0.0747 & -0.0028 & 0.0414 & 14 \\
\hline 6 & 0.1697 & 0.0234 & 0.0052 & 0.1107 & 4 \\
\hline 7 & -0.1186 & 0.0293 & -0.0013 & 0.0822 & 8 \\
\hline 8 & -0.0916 & 0.0654 & -0.0030 & 0.0798 & 10 \\
\hline 9 & -0.0441 & -0.1642 & -0.0011 & 0.0891 & 7 \\
\hline 10 & -0.1225 & -0.0170 & 0.0022 & 0.0799 & 9 \\
\hline 11 & 0.1441 & 0.0537 & 0.0060 & 0.1069 & 5 \\
\hline 12 & -0.0100 & 0.0936 & -0.0022 & 0.0417 & 13 \\
\hline 13 & -0.1111 & -0.1619 & 0.0072 & 0.1284 & 2 \\
\hline 14 & -0.0696 & -0.0102 & -0.0015 & 0.0456 & 12 \\
\hline 15 & -0.0047 & 0.0638 & -0.0017 & 0.0272 & 15 \\
\hline 16 & 0.1788 & 0.0545 & 0.0047 & 0.1280 & 3 \\
\hline
\end{tabular}

this $\gamma$ obtained based on equal weights is used for comparison, which is also enumerated in Table 4.

Then, KPCA was performed on the above $\xi$ array to extract the kernel principal components and identify the corresponding weights. Based on Eqs. (6)-(9), the 
Table 6 Response table for $\gamma$

\begin{tabular}{llllll}
\hline Experimental factors & \multicolumn{2}{l}{ Average $\gamma$} & & Range \\
\cline { 2 - 5 } & Level 1 & Level 2 & Level 3 & Level 4 & 0.0201 \\
\hline Type of inserts $A$ & 0.5355 & $0.5556^{*}$ & & & 3 \\
Feed rate $B$ & $0.7127^{*}$ & 0.5330 & 0.4814 & 0.4552 & 0.2575 \\
Depth of cut $C$ & $0.6573^{*}$ & 0.5020 & 0.5174 & 0.5055 & 0.1553 \\
\hline
\end{tabular}

*Optimum factor level

Table 7 Response table for $\gamma_{k}$

\begin{tabular}{lllllc}
\hline Experimental factors & Average $\gamma_{k}$ & & & Range \\
\cline { 2 - 5 } & Level 1 & Level 2 & Level 3 & Level 4 & \\
\hline Type of inserts $A$ & $0.0886^{*}$ & 0.0808 & & 0.0077 & 3 \\
Feed rate $B$ & $0.1163^{*}$ & 0.0658 & 0.0717 & 0.0851 & 0.0505 \\
Depth of cut $C$ & $0.1379^{*}$ & 0.0344 & 0.0712 & 0.0953 & 0.1036 \\
\hline
\end{tabular}

*optimum factor level

eigenvalues and the corresponding eigenvectors were calculated, and all the eigenvalues were listed in descending order. The bar chart in Fig. 4 shows the contribution rate of each eigenvalue, and the line chart shows the accumulative contribution rate, $\zeta$. It can be clearly observed that the $\zeta$ of the first three eigenvalues reaches up to $99 \%$, which means that the first three kernel principal components hold the most amount of information. Thus, the first three kernel principal components were extracted according to Eq. (10), as shown in Table 5. The corresponding weights for these components obtained from $\zeta$ were $59.87 \%, 38.16 \%$ and $1.98 \%$, respectively. Lastly, the new criterion $\gamma_{k}$ for multiresponse optimization were calculated by Eq. (11), with its results listed in Table 5.

The last columns of Tables 4 and 5 show the rankings of $\gamma$ and $\gamma_{k}$, respectively. According to the previous analysis, a larger $\gamma$ or $\gamma_{k}$ means that the observed sequence is more closely related to the reference sequence representing the best performance. Thus, the largest $\gamma$ and the largest $\gamma_{k}$ correspond to the optimal parameter combinations, respectively. By comparing the ranking results of the last column in Tables 4 and 5, it can be found that among all the tests, the largest $\gamma$ and the largest $\gamma_{k}$ both appear under Run No.1, which means that in the 16 tests studied, the optimal combinations of process parameters determined based on these two methods are consistent. However, in order to find out the relative importance of the process parameters on the integrated optimization criterion, it is necessary to further analyze the effect of each parameter level to identify the optimal combination of process parameters more accurately.

\subsection{Effect of process parameters on the integrated optimization criterion}

To evaluate the effect of process parameters on the integrated optimization criterion, the average $\gamma$ or $\gamma_{k}$ for each parameter level was calculated and listed in the response Tables 6 and 7, respectively. Based on the theory that "the larger the $\gamma$, the better the performance" $[15,20]$, the combination of process parameters corresponding to the maximum average response is optimal.

For $\gamma$ in Table 6 , it can be clearly observed that the combination of process parameters $A_{2}$ (type of inserts: Nose 2), $B_{1}$ (feed rate: $0.1 \mathrm{~mm} / \mathrm{r}$ ), and $C_{1}$ (depth of cut: 0.5 $\mathrm{mm}$ ) presents the largest $\gamma$ with $0.5556,0.7127$, and 0.6573 , respectively. That is to say, $A_{2} B_{1} C_{1}$ is the optimal combination of process parameters for Ti-6Al-4V turning based on this method. According to the results of range analysis listed in the last two columns, feed rate has the most dominant influence on $\gamma$, followed by depth of cut and type of inserts. Figure 5a illustrates the effect of each factor at different levels on $\gamma$. It can be seen that the optimal comprehensive performance can be obtained at low feed rate and low depth of cut with insert Nose 2.

However, in terms of $\gamma_{k}$ in Table 7, the combination of process parameters $A_{1} B_{1} C_{1}$ (type of inserts: Nose 1, feed rate: $0.1 \mathrm{~mm} / \mathrm{r}$, and depth of cut: $0.5 \mathrm{~mm}$ ), has the best performance with the maximum average responses. Furthermore, the results of range analysis reveal that depth of cut is the most significant factor that affects the integrated optimization criterion $\gamma_{k}$. The detailed effects of the process parameters are illustrated in Fig. 5b. Compared with the conclusions drawn from Fig. 5a, it can be inferred that in order to obtain the maximum integrated optimization 


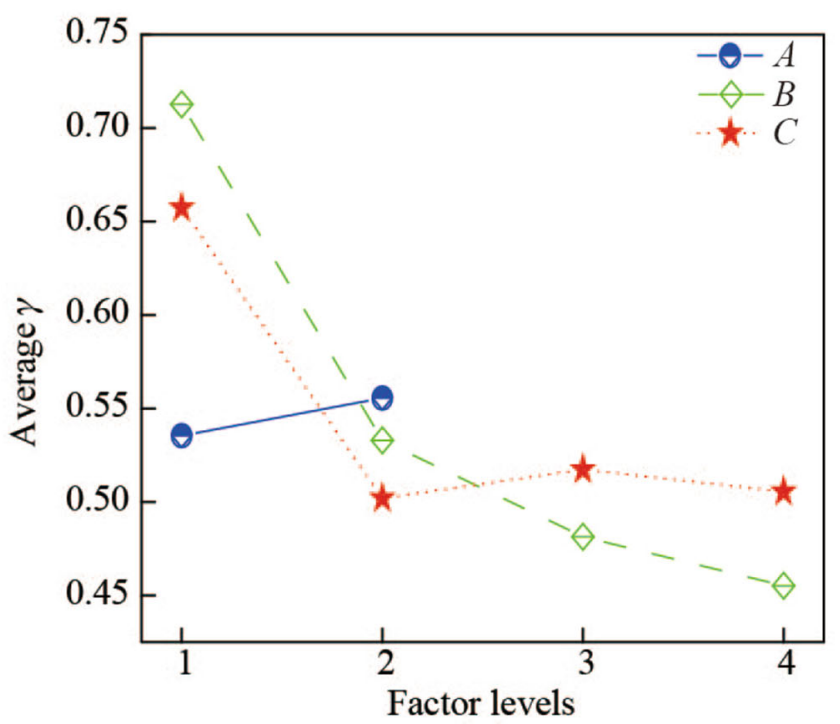

(a)

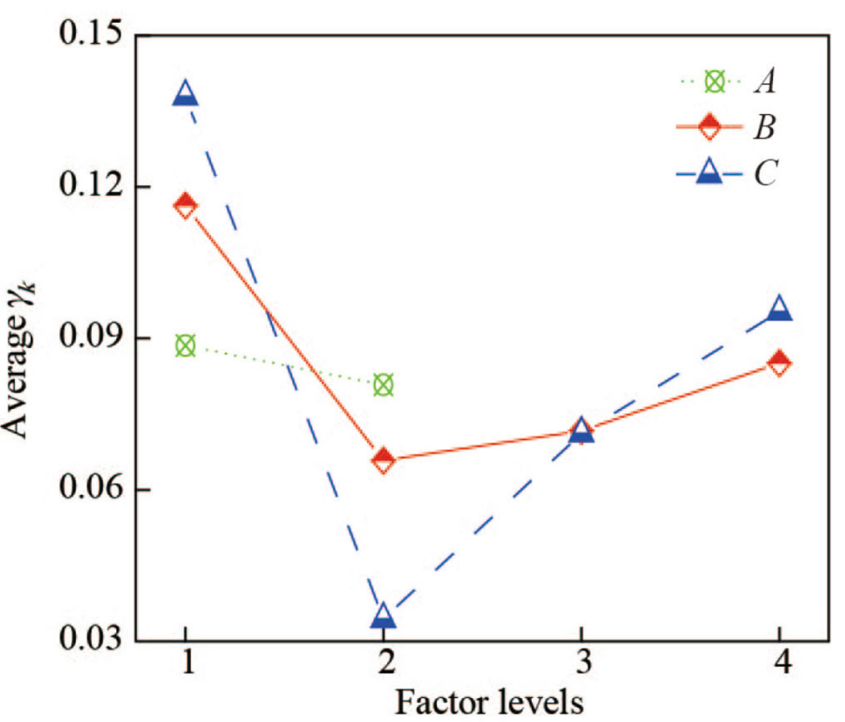

(b)

Fig. 5 Effect of factors at different levels on $\mathbf{a} \gamma$ and $\mathbf{b} \gamma_{k}$

Table 8 Eigenvalues and the corresponding contribution rate for principal components

\begin{tabular}{lll}
\hline Principal components & Eigenvalues & Contribution rate/\% \\
\hline First & 0.0717 & 60.76 \\
Second & 0.0447 & 37.83 \\
Third & 0.0017 & 1.41 \\
\hline
\end{tabular}

Table 9 Eigenvectors for each principal component

\begin{tabular}{|c|c|c|c|}
\hline \multirow{2}{*}{$\begin{array}{l}\text { Performance } \\
\text { index }\end{array}$} & \multicolumn{3}{|l|}{ Eigenvectors } \\
\hline & $\begin{array}{l}\text { First principal } \\
\text { component }\end{array}$ & $\begin{array}{l}\text { Second principal } \\
\text { component }\end{array}$ & $\begin{array}{l}\text { Third principal } \\
\text { component }\end{array}$ \\
\hline Radial thrust & 0.4438 & 0.6965 & -0.5638 \\
\hline $\begin{array}{l}\text { Cutting } \\
\text { power }\end{array}$ & 0.8144 & -0.0510 & 0.5780 \\
\hline $\begin{array}{l}\text { Coefficient } \\
\text { of friction }\end{array}$ & -0.3738 & 0.7157 & 0.5899 \\
\hline
\end{tabular}

Table 10 Contribution rate of each performance index to the fist principal component

\begin{tabular}{lll}
\hline Performance index & Contribution rate/\% & Weight \\
\hline Radial thrust & 19.70 & 0.1970 \\
Cutting power & 66.33 & 0.6633 \\
Coefficient of friction & 13.97 & 0.1397 \\
\hline
\end{tabular}

criterion, Fig. 5b gives a similar opinion in the aspect of depth of cut and feed rate, but different in type of inserts. This may be caused by the nonlinear relationship between the performance indexes. In Fig. 5a, there is an approximate linear relationship, while in Fig. 5b, the nonlinear relationship can be clearly observed. Therefore, the effect of nonlinearity on multi-response optimization results deserves further study.

\subsection{Effect of nonlinearity on multi-response optimization results}

In order to clearly illustrate the effect of nonlinearity among the performance indexes on the multi-response optimization results, the hybrid method based on GRA and PCA by Lu et al. [28] was used for comparison. According to the $\xi$ array in Table 4, PCA was first carried out. The eigenvalues and the corresponding eigenvectors were presented in Tables 8 and 9, respectively. It can be clearly observed from Table 8 that the contribution rate reaches up to $60.76 \%$ when using the first principal component to characterize the original performance indexes, which is very high. Because the square of the value in each eigenvector stands for the corresponding contribution rate of the performance index to each principal component, so according to Lu's method, the square of the value in the first eigenvector is determined as the weight of the corresponding performance index, as shown in Table 10 .

According to Eq. (4) and the GRC array in Table 4, the corresponding grey relational grade $\gamma_{p}$ was calculated, and the corresponding response table was shown in Table 11. It can be observed that by the hybrid method based on GRA and PCA, the obtained optimal combination of process parameters is $A_{2} B_{1} C_{1}$, which is identical to that obtained by the traditional GRA method with equal weights in Table 6, 
Table 11 Response table for $\gamma_{\mathrm{p}}$

\begin{tabular}{lllllc}
\hline Experimental factors & \multicolumn{2}{l}{ Average $\gamma_{\mathrm{p}}$} & & \multicolumn{2}{c}{ Range } \\
\cline { 2 - 5 } & Level 1 & Level 2 & Level 3 & Level 4 & \\
\hline Type of inserts $A$ & 0.5373 & $0.5383^{*}$ & & 0.0010 & 3 \\
Feed rate $B$ & $0.6452^{*}$ & 0.5290 & 0.4930 & 0.4840 & 0.1612 \\
Depth of cut $C$ & $0.7743^{*}$ & 0.5081 & 0.4506 & 0.4181 & 0.3562 \\
\hline
\end{tabular}

*Optimum factor level

Table 12 Comparison between the initial combination and the optimal combinations

\begin{tabular}{lcccc}
\hline Combinations & Symbol & $\begin{array}{l}\text { Radial } \\
\text { thrust }\end{array}$ & $\begin{array}{l}\text { Power } \\
\text { consumption }\end{array}$ & $\begin{array}{l}\text { Coefficient of } \\
\text { friction }\end{array}$ \\
\hline Initial & $A_{2} B_{2} C_{2}$ & 204.17 & 206.51 & 2.21 \\
$\begin{array}{l}\text { Optimized by } \\
A_{2} B_{1} C_{1}\end{array}$ & 163.12 & 88.03 & 2.17 \\
$\quad \gamma / \gamma_{\mathrm{p}}$ & & & & \\
$\begin{array}{l}\text { Relative increment } \\
\text { Optimized by }\end{array}$ & $A_{1} B_{1} C_{1}$ & $-20.11 \%$ & $-57.37 \%$ & $-1.67 \%$ \\
$\quad \gamma_{k}$ & & 85.09 & 2.20 \\
$\begin{array}{l}\text { Relative increment } \\
\text { n }\end{array}$ & $-21.59 \%$ & $-58.45 \%$ & $-0.25 \%$ \\
\hline
\end{tabular}

but different from the optimal result obtained by the proposed method in Table 7. The difference between the optimization results is exactly caused by the nonlinearity among the performance indexes. Furthermore, the depth of cut is the most significant factor for $\gamma_{\mathrm{p}}$, which is the same with that for $\gamma_{k}$.

In order to demonstrate the effectiveness of the proposed method, the obtained optimization results need further verification. The verification tests and comparison results were presented in Sect. 3.4.

\subsection{Verification tests}

In this paper, the verification tests were conducted under the conditions of the initial process combination $A_{2} B_{2} C_{2}$ (type of inserts: Nose 2, feed rate: $0.15 \mathrm{~mm} / \mathrm{r}$, and depth of cut: $1.0 \mathrm{~mm}$ ), and the above two optimal combinations $A_{2} B_{1} C_{1}$ and $A_{1} B_{1} C_{1}$ in order to demonstrate the effectiveness of the proposed method. The comparison results and error graphs of the performance indexes $F_{y}, P$, and $\mu$ in these tests were presented in Table 12 and Fig. 6, respectively.

It can be seen that relative to the initial condition, there is a clear improvement in terms of radial thrust and power consumption, and a minor improvement in the coefficient of friction for both of the optimization results. As illustrated in Table 12, under the optimal combination $A_{1} B_{1} C_{1}$ obtained by $\gamma_{k}$, the power consumption is reduced by $58.45 \%$, and the radial thrust is decreased by $21.59 \%$, which is superior to those under the optimal combination $A_{2} B_{1} C_{1}$ obtained by $\gamma$ and $\gamma_{p}$, though there is little difference in the coefficient of friction. Consequently, the results of these tests reveal that the modified method combining GRA with KPCA is very effective for solving multi-response optimization problems and outperforms the singleGRA method with equal weights and the hybrid method based on GRA and PCA.

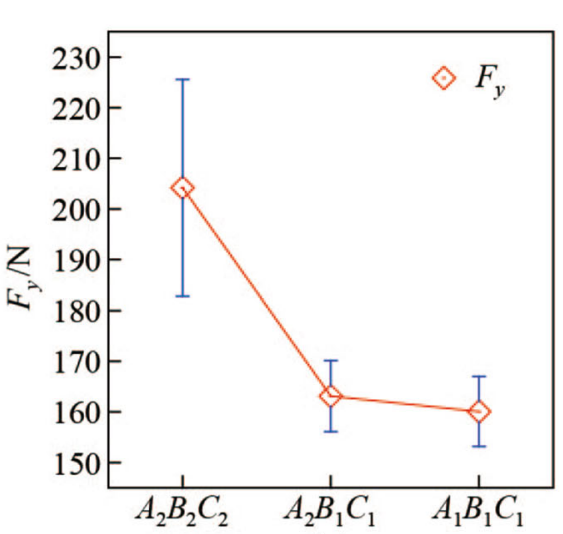

(a)

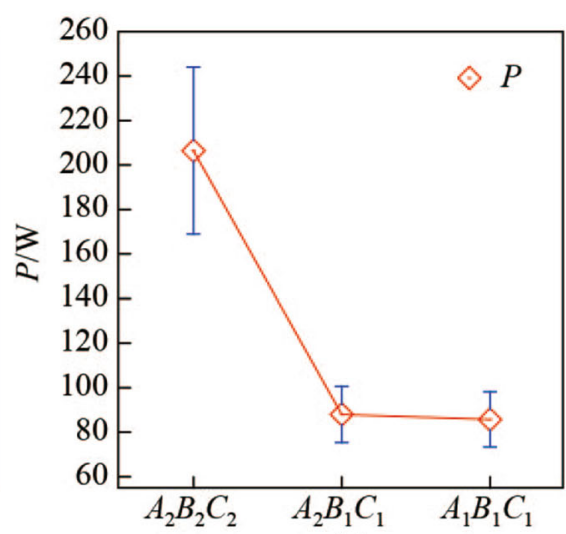

(b)

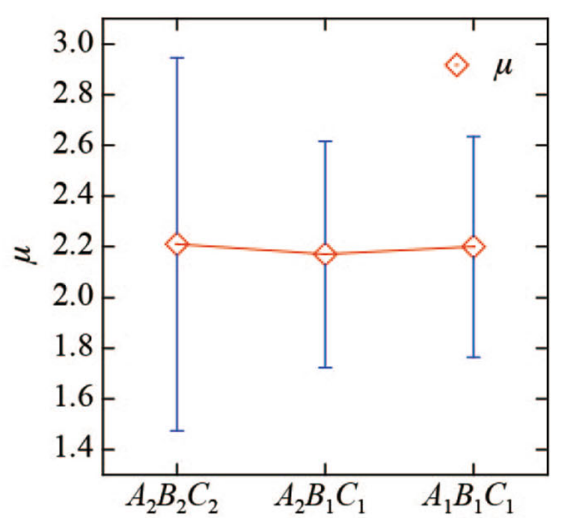

(c)

Fig. 6 Error graph of $\mathbf{a} F_{y}, \mathbf{b} P$, and $\mathbf{c} \mu$ 


\section{Conclusions}

Taken the nonlinearity of cutting process into consideration, a new method based on GRA and KPCA was proposed for the multi-response optimization problem during turning of Ti-6Al-4V. The radial thrust, power consumption, and coefficient of friction at the tool-chip interface were chosen as the performance indexes. Compared with the traditional method based on GRA with equal weights and the hybrid method based on GRA and PCA, the following conclusions can be drawn

(i) KPCA was performed to extract the kernel principal components and identify the corresponding weights. The first three kernel principal components were extracted and the corresponding weights obtained from $\zeta$ were $59.87 \%, 38.16 \%$, and $1.98 \%$, respectively.

(ii) Within the range of process parameters studied, the largest $\gamma$ and $\gamma_{k}$ both appeared in Run No. 1 .

(iii) For $\gamma$ and $\gamma_{\mathrm{p}}, A_{2} B_{1} C_{1}$ is the optimal combination of process parameters, while for $\gamma_{k}$, the combination $A_{1} B_{1} C_{1}$ is the best.

(iv) Feed rate has the most dominant effect on $\gamma$, followed by depth of cut and type of insert, while whereas for $\gamma_{p}$ and $\gamma_{k}$, the depth of cut is the most significant factor. A consistent view is that the optimal comprehensive performance can be obtained at a low feed rate and low depth of cut.

(v) The comparison results show that the nonlinearity in the multi-response optimization problem cannot be neglected.

(vi) Verification tests reveal that there is a clear improvement in terms of radial thrust and power consumption, and a minor improvement in the coefficient of friction at the tool-chip interface for both of the optimization results, but the proposed method outperforms the single-GRA method with equal weights and the hybrid method based on GRA and PCA.

Acknowledgements The authors would like to acknowledge the financial assistance from the National Science and Technology Major Project of China (Grant No. 2012ZX04003-021).

Open Access This article is distributed under the terms of the Creative Commons Attribution 4.0 International License (http://crea tivecommons.org/licenses/by/4.0/), which permits unrestricted use, distribution, and reproduction in any medium, provided you give appropriate credit to the original author(s) and the source, provide a link to the Creative Commons license, and indicate if changes were made.

\section{References}

1. Zhou L, Zhao YQ, Wang XD (2012) Study on development strategy of titanium alloy materials and application in China. Chemical Industry Press, Beijing

2. Çolak O (2014) Optimization of machining performance in highpressure assisted turning of Ti6Al4V alloy. Stroj Vestn-J Mech Eng 60(10):675-681

3. Chinchanikar S, Choudhury SK (2013) Effect of work material hardness and cutting parameters on performance of coated carbide tool when turning hardened steel: an optimization approach. Measurement 46:1572-1584

4. Amin AKMN, Ismail AF, Khairusshima MKN (2007) Effectiveness of uncoated WC-Co and PCD inserts in end milling of titanium alloy-Ti-6Al-4V. J Mater Process Technol 192(5):147-158

5. Sun FJ, Qu SG, Pan YX et al (2015) Effects of cutting parameters on dry machining Ti-6Al-4V alloy with ultra-hard tools. Int J Adv Manuf Technol 79(1-4):351-360

6. Wu KZ, Chen YJ, Zhu DD et al (2005) Application of frictionreducing groove on insert with $3 \mathrm{D}$ chip-breaking groove. Tool Technol 39(5):53-55

7. Arulkirubakaran D, Senthilkumar V, Kumawat V (2016) Effect of micro-textured tools on machining of Ti-6Al-4V alloy: an experimental and numerical approach. Int $\mathrm{J}$ Refract Met Hard Mater 54:165-177

8. Li N, Chen YJ, Kong DD et al (2017) Experimental investigation with respect to the performance of deep submillimeter-scaled textured tools in dry turning titanium alloy Ti-6Al-4V. Appl Surf Sci 403:187-199

9. Pusavec F, Krajnik P, Kopac J (2010) Transitioning to sustainable production-Part I: application on machining technologies. J Clean Prod 18(2):174-184

10. Pusavec F, Krajnik P, Kopac J (2010) Transitioning to sustainable production-Part II: evaluation of sustainable machining technologies. J Clean Prod 18(12):1211-1221

11. Malakooti B, Wang J, Tandler EC (1990) A sensor-based accelerated approach for multi-attribute machinability and tool life evaluation. Int J Prod Res 28(12):2373-2392

12. Subramanian M, Sakthivel M, Sooryaprakash K et al (2013) Optimization of end mill tool geometry parameters for A17075T6 machining operations based on vibration amplitude by response surface methodology. Measurement 46(10):4005-4022

13. Singh D, Rao PV (2007) Optimization of tool geometry and cutting parameters for hard turning. Mater Manuf Process 22(1):15-21

14. Ramana MV, Rao GKM, Rao DH (2014) Optimization and effect of process parameters on tool wear in turning of titanium alloy under different machining conditions. Int J Mater Mech Manuf 2(4):272-277

15. Yan JH, Li L (2013) Multi-objective optimization of milling parameters - the trade-offs between energy, production rate and cutting quality. J Clean Prod 52:462-471

16. Sarıaya M, Güllü A (2015) Multi-response optimization of minimum quantity lubrication parameters using Taguchi-based grey relational analysis in turning of difficult-to-cut alloy Haynes 25. J Clean Prod 91:347-357

17. Thepsonthi T, Özel T (2012) Multi-objective process optimization for micro-end milling of Ti-6Al-4V titanium alloy. Int J Adv Manuf Technol 63(9-12):903-914

18. Yi Q, Li CB, Tang Y et al (2015) Multi-objective parameter optimization of CNC machining for low carbon manufacturing. J Clean Prod 95:256-264

19. Nayak SK, Patro JK, Dewangan S et al (2014) Multi-objective optimization of machining parameters during dry turning of AISI 
304 austenitic stainless steel using grey relational analysis. Procedia Mater Sci 6:701-708

20. Mia M, Khan MA, Rahman SS et al (2017) Mono-objective and multi-objective optimization of performance parameters in high pressure coolant assisted turning of Ti-6Al-4V. Int J Adv Manuf Technol 90(1-4):109-118

21. Mia M, Khan MA, Dhar NR (2017) Study of surface roughness and cutting forces using ANN, RSM, and ANOVA in turning of Ti-6Al-4V under cryogenic jets applied at flank and rake faces of coated WC tool. Int J Adv Manuf Technol 93(1-4):975-991

22. Xiong LS, Yan XG, Zhang FR (2006) Fundamentals of mechanical manufacturing technology. Huazhong University of Science and Technology Press, Wuhan

23. Newman ST, Nassehi A, Asrai RI et al (2012) Energy efficient process planning for CNC machining. CIRP J Manuf Sci Technol 5(2): $127-136$

24. Mia M, Rifat A, Tanvir MF et al (2018) Multi-objective optimization of chip-tool interaction parameters using grey-Taguchi method in MQL-assisted turning. Measurement 129:156-166

25. Zhang TY, Owodunni O, Gao J (2015) Scenarios in multi-objective optimization of process parameters for sustainable machining. Procedia CIRP 26:373-378
26. Hanafi I, Khamlichi A, Cabrera FM et al (2012) Optimization of cutting conditions for sustainable machining of PEEK-CF30 using TiN tools. J Clean Prod 33:1-9

27. Bhushan RK (2013) Optimization of cutting parameters for minimizing power consumption and maximizing tool life during machining of $\mathrm{Al}$ alloy $\mathrm{SiC}$ particle composites. J Clean Prod 39:242-254

28. Lu HS, Chang CK, Hwang NC et al (2009) Grey relational analysis coupled with principal component analysis for optimization design of the cutting parameters in high-speed end milling. J Mater Process Technol 209:3808-3817

29. Dubey AK, Yadava V (2008) Multi-objective optimization of Nd:YAG laser cutting of nickel-based superalloy sheet using orthogonal array with principal component analysis. Opt Lasers Eng 46(2):124-132

30. Ross PJ (1988) Taguchi techniques for quality engineering. McGraw-Hill, New York

31. Deng JL (1982) The course of grey system theory. Huazhong University of Science and Technology Press, Wuhan 